\title{
miR-142-5p inhibits pancreatic cancer cell migration and invasion by targeting PIK3CA
}

\author{
JING ZHU, LEILEI ZHOU, BIN WEI，ZHAOYE QIAN，JIRU WANG，HONGXIA HUI and YUAN SUN \\ Department of Medical Oncology, The Affiliated Huaian No. 1 People's Hospital \\ of Nanjing Medical University, Huai'an, Jiangsu 223300, P.R. China
}

Received February 13, 2019; Accepted September 20, 2019

DOI: $10.3892 / \mathrm{mmr} .2020 .11251$

\begin{abstract}
Pancreatic cancer is a fatal disease with a high mortality rate and poor prognosis worldwide. The aberrant expression of microRNAs (miRs) is associated with cancer development and progression. The present study aimed to evaluate the functional role of miR-142-5p in migration and invasion, and investigated its underlying molecular mechanism in pancreatic cancer cells. First, it was identified that miR-142-5p expression was downregulated in pancreatic cancer tissues and cell lines by reverse transcription-quantitative polymerase chain reaction. Furthermore, phosphoinositide-3-kinase catalytic subunit $\alpha$ (PIK3CA) was identified as a target of miR-142-5p. The expression of PIK3CA was upregulated in tumor tissues and its expression was negatively regulated by miR-142-5p expression. Notably, overexpression of miR-142-5p inhibited the proliferation, migration and invasion of PanC1 cells, while PIK3CA reversed this inhibition. In addition, miR-142-5p suppressed the expression of focal adhesion kinase (FAK) and matrix metalloproteinase (MMP)9, as well as phosphorylated protein kinase B (AKT) protein level, while PIK3CA reversed the suppression induced by miR-142-5p. In conclusion, miR-142-5p functions as a tumor suppressor, which inhibits the migration and invasion of pancreatic cancer by suppressing the expression of FAK and MMP9, as well as the phosphatidylinositol 3-kinase/AKT signaling pathway by targeting PIK3CA. These findings suggest that miR-142-5p may be a novel therapeutic target for the treatment of pancreatic cancer.
\end{abstract}

\section{Introduction}

Pancreatic cancer is a highly lethal disease with a mortality rate that is similar to the incidence rate and is a worldwide health

Correspondence to: Dr Yuan Sun, Department of Medical Oncology, The Affiliated Huaian No. 1 People's Hospital of Nanjing Medical University, 6 Beijing West Road, Huaiyin, Huai'an, Jiangsu 223300, P.R. China

E-mail: sunyuan2019@yandex.com

Key words: miR-142-5p, phosphoinositide-3-kinase catalytic subunit $\alpha$, pancreatic cancer, migration, invasion burden, particularly in China $(1,2)$. Pancreatic cancer accounts for $5 \%$ of all cancer-associated mortality (3). In addition, the prognosis of pancreatic cancer is very poor, with an overall 5 -year survival rate of only $\sim 5 \%$ (4) and a median survival time of $\leq 6$ months (5). Despite developments in surgical treatment and early detection, this situation has not significantly changed $(6,7)$. The poor prognosis is mainly due to the fact that nearly $80 \%$ of patients present with locally advanced or metastatic disease (8). Therefore, improved understanding regarding the molecular mechanisms involved in pancreatic cancer will be helpful for providing new insights into effective treatments for patients with pancreatic cancer.

MicroRNAs (miRNAs/miRs) are a class of small, non-coding, regulatory RNAs that are 22 nucleotides in length (9). Currently, >2,000 miRNAs have been discovered in humans, which regulate one third of all genes (10). miRNAs regulate the expression of their target protein-coding genes by degradation of mRNA or translational inhibition through binding to the 3'-untranslated regions (3'-UTRs) of target genes (11). Dysregulation of miRNAs is common in various human cancer types, with upregulated miRNAs often acting as oncogenes and downregulated miRNAs acting as tumor suppressors (12). These cancer-associated miRNAs mediate various biological functions associated with cancer development and progression, including proliferation, differentiation, cell signaling, cell survival, apoptosis and metastasis (13). Due to their small size and the network of proteins regulated by miRNAs, miRNA therapeutics is developing; therefore, more miRNAs need to be identified that could be applied in clinical trials (14). miR-142 serves a critical role in cancer, virus infection, inflammation and immune tolerance, and miR-142-5p is the passenger strand generated from the miR-142 hairpin (15). A previous study revealed that overexpression of miR-142-5p could inhibit tumor growth of pancreatic cancer in vivo (16). However, the biological function and molecular mechanism of miR-142-5p in pancreatic cancer remain largely unknown.

The present study measured the expression of miR-142-5p in pancreatic cancer tissues and cell lines. Additionally, the effects of miR-142-5p, as well as its target gene phosphoinositide-3-kinase catalytic subunit $\alpha$ (PIK3CA), on cell proliferation, migration and invasion were investigated. The results demonstrated that miR-142-5p inhibits the migration and invasion by targeting PIK3CA, which suggests that 
miR-142-5p may be a potential target for the treatment of pancreatic cancer.

\section{Materials and methods}

Patients and specimens. Pancreatic cancer tissue and paired adjacent normal tissue samples $(n=35$, including 24 male and 11 female; age range from 48-77 years and the average age was 62.17 years) were obtained from patients who underwent surgery at The Affiliated Huaian No.1 People's Hospital of Nanjing Medical University (Huai'an, China) from February 2017 to July 2018. All patients received no preoperative treatment and provided written informed consent prior to surgery. The fresh tissues were immediately frozen in liquid nitrogen and stored at $-80^{\circ} \mathrm{C}$ until further use. The present study was approved by the Ethics Committee of The Affiliated Huaian No.1 People's Hospital of Nanjing Medical University.

Cell lines and culture. Human pancreatic cancer cell lines (PanC1, BxPC3, SW1990 and CAPAN-1) and the normal human fibroblast WI-38 cell line were purchased from the Cell Bank of Type Culture Collection of the Chinese Academy of Sciences. All cells were cultured in Dulbecco's modified Eagle's medium (DMEM; Gibco; Thermo Fisher Scientific, Inc.) supplemented with $10 \%$ fetal bovine serum, $100 \mathrm{U} / \mathrm{ml}$ penicillin and $100 \mu \mathrm{g} / \mathrm{ml}$ streptomycin (all from Gibco; Thermo Fisher Scientific, Inc.), and incubated in an incubator at $37^{\circ} \mathrm{C}$ with $5 \% \mathrm{CO}_{2}$.

Prediction of miR-124-5p target genes. The miR-142-5p targets were predicted using Targetscan (http://www.targetscan. org/vert_72/).

Dual-luciferase reporter assay. A pGL3 plasmid was purchased from Promega Corporation. The wild-type (WT) or mutated 3'-UTR of PIK3CA was synthesized and cloned into pGL3 downstream of the firefly luciferase reporter. PanC1 cells were seeded in 96-well plates until the confluence reached $70 \%$. Subsequently, the cells were co-transfected with WT or mutant 3'-UTR reporter vector and miR-negative control (NC) or miR-142-5p mimic using Lipofectamine ${ }^{\circledR} 2000$ (Invitrogen; Thermo Fisher Scientific, Inc.), according to manufacturer's protocol. At $48 \mathrm{~h}$ post-transfection, firefly and Renilla luciferase activities were measured using the Dual-Luciferase Reporter assay system (Promega Corporation). The Renilla luciferase activity was used for endogenous normalization.

Celltransfection.miR-142-5pmimic (cat.no.miR10000433-1-5; sense, 5'-CGUGUUCACAGCGGACCUUGAU-3' and anti-sense, 5'-AUCAAG GUCCGCUGUGAACACG-3') and the corresponding NC (cat. no. miR1N0000002-1-5; sense, 5'-UAGCCAUAUCGUCGAUACU-3' and anti-sense, 5'-AGUAUCGACGAUAUGGCUA-3') were purchased from Guangzhou RiboBio Co., Ltd. pcDNA3.1 was obtained from Invitrogen; Thermo Fisher Scientific, Inc. The coding region of PIK3CA was inserted into the pcDNA3.1 vector and termed pcDNA3.1-PIK3CA. PanC1 cells were seeded into six-well plates at a density of $4 \times 10^{5}$ cells/well $24 \mathrm{~h}$ prior to transfection. Subsequently, the cells were transfected with $20 \mathrm{nM} \mathrm{miR}-124-5 \mathrm{p}$ mimic, miR-NC, pcDNA3.1 or pcDNA3.1-PIK3CA using
Lipofectamine ${ }^{\circledR} 2000$ (Invitrogen; Thermo Fisher Scientific, Inc.) in serum-free DMEM, according to the manufacturer's protocol. At $6 \mathrm{~h}$ post-transfection, the cells were incubated with complete medium. After $48 \mathrm{~h}$ of transfection, the cells were harvested for further study. Untransfected cells were used as the control group.

Reverse transcription-quantitative PCR (RT-qPCR). Total RNA was extracted from paired pancreatic cancer tissues, corresponding normal tissues and $\mathrm{PanC} 1$ cells using the TRIzol $^{\circledR}$ reagent (Invitrogen, Thermo Fisher Scientific, Inc.), according to the manufacturer's protocol. Next, the total RNA was reverse transcribed to cDNA at $60^{\circ} \mathrm{C}$ for $30 \mathrm{~min}$ using the ThermoScript RT-PCR system (Invitrogen; Thermo Fisher Scientific, Inc.). Subsequently, qPCR was performed using the All-in-One ${ }^{\mathrm{TM}}$ miRNA RT-qPCR detection kit (GeneCopoeia, Inc.) to detect miR-142-5p expression and using SYBR Green PCR Mastermix (Beijing Solarbio \& Technology, Co., Ltd.) to detect the mRNA expression. All reactions were performed on an ABI PRISM 7500 Fast Real-time PCR system (Applied Biosystems; Thermo Fisher Scientific, Inc.). The qPCR amplification conditions consisted of $95^{\circ} \mathrm{C}$ for $10 \mathrm{~min}$, followed by 40 cycles of $95^{\circ} \mathrm{C}$ for $10 \mathrm{sec}, 60^{\circ} \mathrm{C}$ for $20 \mathrm{sec}$ and $72^{\circ} \mathrm{C}$ for $15 \mathrm{sec}$. The primers sequences were as follows: miR-142-5p F: 5'-AACTCCAGCTGGTCCTTAG-3', R: 5'-TCTTGAACC CTCATCCTGT-3'; small nuclear RNA U6 F: 5'-CTCGCT TCGGCAGCACA-3', R: 5'-AACGCTTCACGAATTTGC GT-3'; PIK3CA F: 5'-AAATGAAAGCTCACTCTGGAT TCC-3', R: 5'-TTGTGCAATTCCTATGCAATCG-3'; FAK F: 5'-GTGCTCTTGGTTCAAGCTGGAT-3', R: 5'-ACTTGA GTGAAGTCAGCAAGATGTGT-3'; MMP9 F: 5'-GTGCTG GGCTGCTGCTTTGCTG-3', R: 5'-GTCGCCCTCAAAGGT TTGGAAT-3'; AKT F: 5'-AGGCATCCCTTCCTTACAGC-3', R: 5'-CAGCCCGAAGTCCGTTATCT-3'; GAPDH F: 5'-GAA GGTGAAGGTCGGAGTC-3', R: 5'-GAAGATGGTGATGGG ATTTC-3'. U6 and GAPDH were used as the loading control for miR-142-5p and mRNA, respectively. The relative expression was calculated using the $2^{-\Delta \Delta C q}$ method (17).

Western blotting. The harvested PanC1 cells were lysed using RIPA buffer (Beyotime Institute of Biotechnology) on ice for $30 \mathrm{~min}$. The Bicinchoninic Acid Protein assay kit (Beyotime Institute of Biotechnology) was used to detect the protein concentration. Subsequently, $30 \mu \mathrm{g}$ protein was separated by $10 \%$ SDS-PAGE and transferred onto PVDF membranes (EMD Millipore). After blocking at room temperature for $1 \mathrm{~h}$ with TBST (containing 0.05\% Tween-20; Beijing Solarbio Science \& Technology Co., Ltd.) containing 5\% skim milk, the membranes were incubated with primary antibodies all purchased from Abcam: Anti-PIK3CA (cat. no. ab40776; 1:5,000); anti-FAK (cat. no. ab40794; 1:1,000); anti-MMP9 (cat. no. ab38898; 1:1,000); anti-AKT (cat. no. ab227100; 1:1,000); anti-phosphorylated (p)-AKT (cat. no. ab81283; 1:5,000); anti-GAPDH (cat. no. ab181602; 1:10,000) overnight at $4{ }^{\circ} \mathrm{C}$. The next day, secondary antibody [goat anti-rabbit IgG H\&L (horseradish peroxidase); cat. no. ab6721; 1:10,000; Abcam] was incubated with the membranes at room temperature for $1 \mathrm{~h}$. Finally, the membranes were incubated with BeyoECL Plus (Beyotime Institute of Biotechnology) and the protein bands were visualized. The relative expression was 
calculated using ImageQuant LAS 4010 Imaging system (GE Healthcare Life Sciences). GAPDH was used as an internal control.

Cell proliferation assay. For the cell proliferation assay, a CCK-8 (BiotechWell) assay was performed according to the manufacturer's protocols. Briefly, $2 \times 10^{3}$ transfected PanC1 cells were seeded into 96 -well plates and cultured in an incubator for $0,12,24$ and $48 \mathrm{~h}$. At the indicated time points, $10 \mu \mathrm{l}$ CCK-8 solution was added to each well and the cells were further incubated for $1 \mathrm{~h}$. The absorbance was measured at $450 \mathrm{~nm}$ using a microplate reader (Thermo Fisher Scientific, Inc.).

Cell migration assay. A wound healing assay was performed to analyze cell migration ability. Transfected cells were seeded into 6-well plates at a density of $2 \times 10^{6}$ cells/well. After incubation for $24 \mathrm{~h}$, cell layers were scratched with a $10 \mu \mathrm{l}$-sterile plastic tip and cell debris was rinsed with PBS. The cells were then incubated in serum-free medium at $37^{\circ} \mathrm{C}$ with $5 \% \mathrm{CO}_{2}$ for $24 \mathrm{~h}$. Finally, wound closure was imaged under a light microscope (magnification, x100; Olympus Corporation) at 0 and $24 \mathrm{~h}$ after wounding, and the width was quantified using a standard caliper.

Cell invasion assay. Cell invasion was assessed by Transwell assay using 24-well Matrigel invasion chambers $(8-\mu \mathrm{m}$ pores; EMD Millipore). A total of $1 \times 10^{5}$ cells were added to the upper chambers of Transwell plates filled with serum-free DMEM and complete DMEM added to the bottom chambers. The cells were incubated at $37^{\circ} \mathrm{C}$ with $5 \% \mathrm{CO}_{2}$ for $24 \mathrm{~h}$ and the non-invading cells were then removed with sterile swabs. The invaded cells were fixed with $4 \%$ paraformaldehyde for $10 \mathrm{~min}$ at room temperature, followed by staining with $0.1 \%$ crystal violet for $15 \mathrm{~min}$ at room temperature. The cells were then photographed and quantified in five random fields using an optical light microscope (magnification, x200; Olympus Corporation).

Statistical analysis. Each independent experiment was repeated a minimum of three times. Statistical analysis was performed using GraphPad Prism software (version 6.0; GraphPad Software, Inc.). An unpaired student's t-test was used to evaluate the significant differences between two groups in all cases except a paired student's t-test was used to compare differences between tumor tissues and adjacent normal tissues. A one-way analysis of variance followed by Newman-Keuls post hoc test was used to measure the significant differences among multiple groups. Data are presented as the mean \pm standard error of mean. $\mathrm{P}<0.05$ was considered to indicate a statistically significant difference.

\section{Results}

miR-142-5p is downregulated in pancreatic cancer tissues and cell lines. To determine the expression level of miR-142-5p, RT-qPCR was performed to detect miR-142-5p expression in 35 pairs of pancreatic cancer and matched adjacent normal tissues. Compared with that in adjacent non-tumor tissues, the expression of miR-142-5p was significantly decreased in pancreatic cancer tissues $(\mathrm{P}<0.01$; Fig. 1A). In addition, miR-142-5p expression was significantly downregulated in four pancreatic cancer cell lines (PanC1, BxPC3, SW1990 and CAPAN-1) compared with normal human fibroblast WI-38 cells $(\mathrm{P}<0.01$; Fig. 1B). These results suggested that low miR-142-5p expression may be closely associated with pancreatic cancer progression.

PIK3CA is a target of miR-142-5p. To understand the molecular mechanism underlying miR-142-5p, bioinformatics analysis was performed to identify the potential targets of miR-142-5p. According to the TargetScan database, miR-142-5p was identified to be able to bind to the 3'-UTR of PIK3CA at position 1,675-1,682 nt (Fig. 2A). To further confirm this prediction, cells were co-transfected with WT or mutant 3'-UTR reporter vector as well as miR-NC or miR-142-5p mimic to perform a dual-luciferase reporter assay. As presented in Fig. 2B, overexpression of miR-142-5p significantly decreased the luciferase activity of the plasmid carrying the WT 3'-UTR of PIK3CA in PanC1 cells $(\mathrm{P}<0.01)$. However, miR-142-5p did not affect the luciferase activity when the 3'-UTR of PIK3CA was mutated. Additionally, PanC1 cells were transfected with miR-NC, miR-142-5p mimic, pcDNA3.1 and pcDNA3.1-PIK3CA. miR-142-5p expression was significantly upregulated when transfected with miR-142-5p mimic compared with miR-NC $(\mathrm{P}<0.01)$ and PIK3CA mRNA and protein expression levels were also significantly upregulated when transfected with pcDNA3.1-PIK3CA compared with pcDNA3.1 $(\mathrm{P}<0.01$; Fig. 2C-E). Furthermore, RT-qPCR and western blotting results demonstrated that overexpression of miR-142-5p resulted in a suppression of PIK3CA mRNA and protein levels in PanC1 cells $(\mathrm{P}<0.01$; Fig. $2 \mathrm{~F}$ and $\mathrm{G})$. These results indicated that PIK3CA expression is negatively regulated by miR-142-5p. In summary, PIK3CA was identified as a target of miR-142-5p.

PIK3CA expression is upregulated in pancreatic cancer tissues. Subsequently, the expression of PIK3CA was examined in clinical tissue samples. The mRNA and protein expression levels of PIK3CA were increased in pancreatic cancer tissues compared with matched adjacent normal tissues $(\mathrm{P}<0.01$; Fig. 3A and B). Furthermore, an inverse association was identified between miR-142-5p and PIK3CA expression $(\mathrm{P}=0.0001$; Fig. 3C). These results suggested that the upregulation of PIK $3 \mathrm{CA}$ is partly caused by a downregulation of miR-142-5p and further supports the conclusion that PIK3CA is a target of miR-142-5p.

miR-142-5p inhibits cell proliferation, migration and invasion of pancreatic cancer in vitro by targeting PIK3CA. To assess the role of miR-142-5p in pancreatic cancer, CCK-8, wound healing and Transwell assays were performed to measure the capability of cell proliferation, migration and invasion, respectively. The results demonstrated that overexpression of miR-142-5p significantly inhibited cell proliferation after $48 \mathrm{~h}$ of transfection $(\mathrm{P}<0.01)$, while PIK3CA reversed the inhibition induced by miR-142-5p $(\mathrm{P}<0.01$; Fig. 4A). Furthermore, miR-142-5p-overexpression led to slower wound closure in PanC1 cells compared with the miR-NC group $(\mathrm{P}<0.01)$, while PIK3CA-overexpression significantly reversed the slow closure compared with the 
A

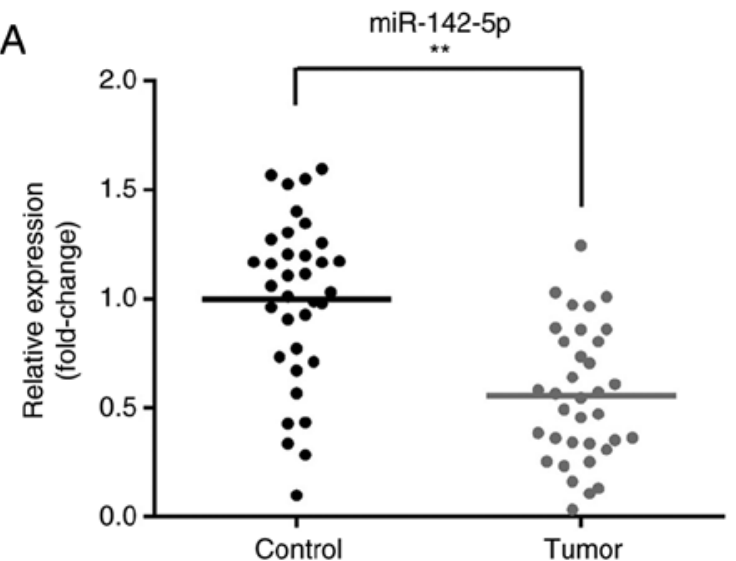

B

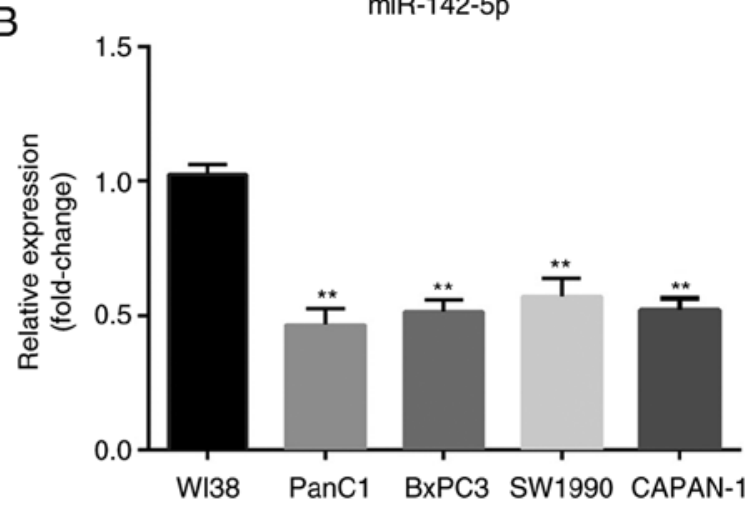

Figure 1. Reduced expression of miR-142-5p in pancreatic cancer tissues and cell lines. (A) RT-qPCR analysis was conducted to measure the expression of miR-142-5p in pancreatic cancer tissues and corresponding non-tumor tissues. (B) miR-142-5p expression in four pancreatic cancer cell lines (PanC1, BxPC3, SW1990 and CAPAN-1) and a normal human fibroblast WI-38 cell line was examined using RT-qPCR. Data are presented as the mean \pm standard error of the mean. ${ }^{* *} \mathrm{P}<0.01$. RT-qPCR, reverse transcription-quantitative PCR; miR, microRNA.

\section{A}

Position 1675-1682 of PIK3CA 3'UTR $55^{\prime}$...UAUUAUUGGAAAACAACUUUAUA...

|||||||

hsa-miR-142-5p $3^{\prime} \quad$ UCAUCACGAAAGAUGAAAUAC

Mutant position of PIK3CA $3^{\prime}$ UTR $55^{\prime}$...UAUUAUUGGAAAACAGCACCGCA...

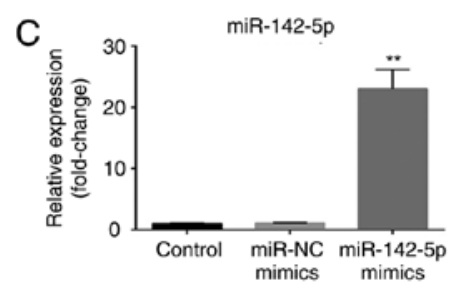

D

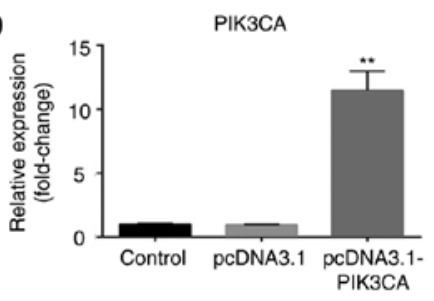

E
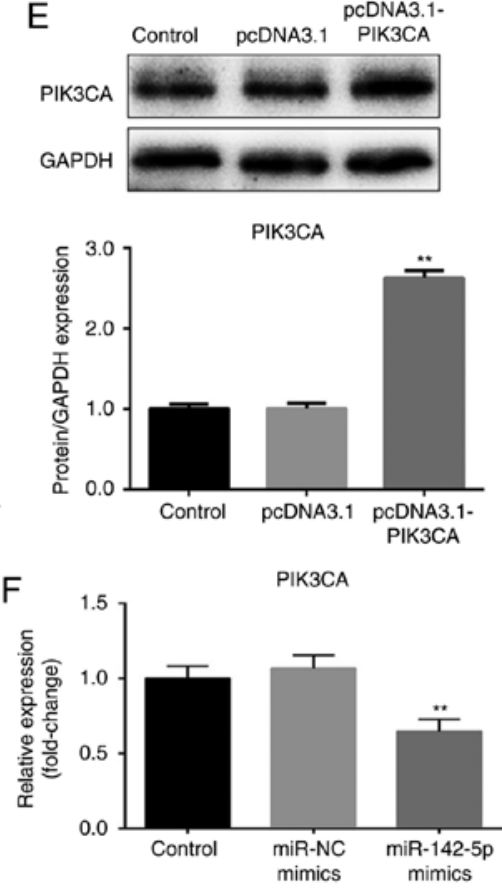

B

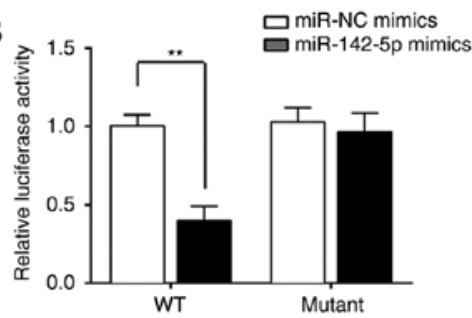

G
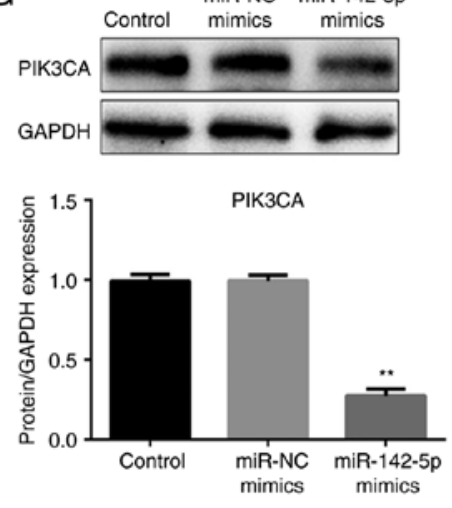

Figure 2. PIK3CA is a target of miR-142-5p in pancreatic cancer cells. (A) Bioinformatics analysis predicted WT and mutant binding sites of miR-142-5p in the 3'-UTR of PIK3CA. (B) Dual-luciferase reporter assay confirmed PIK3CA is a target of miR-142-5p. Luciferase activity was detected after PanC1 cells were co-transfected with WT or mutant PIK3CA 3'-UTR and miR-142-5p mimics and miR-NC mimics. (C) Expression of miR-142-5p in PanC1 cells after transfection with miR-142-5p mimics and miR-NC mimics. (D) PIK3CA expression was measured by reverse transcription-quantitative PCR after PanC1 cells were transfected with pcDNA3.1 and pcDNA3.1-PIK3CA. (E) Protein expression of PIK3CA was measured by western blotting after PanC1 cells were transfected with pcDNA3.1 and pcDNA3.1-PIK3CA. The (F) mRNA and (G) protein expression of PIK3CA in PanC1 cells transfected with miR-142-5p mimics or miR-NC mimics. GAPDH was used as a loading control. Data are presented as the mean \pm standard error of the mean. ${ }^{* *} \mathrm{P}<0.01 \mathrm{vs.}$ miR-NC mimics or pcDNA3.1 group. UTR, untranslated region; WT, wild-type; NC, negative control; miR, microRNA.

miR-142-5p mimic group ( $\mathrm{P}<0.01$; Fig. 4B). Similarly, the invasion ability of PanC1 cells was significantly inhibited by
miR-142-5p mimic $(\mathrm{P}<0.01)$, which was restored by PIK3CA $(\mathrm{P}<0.01 ;$ Fig. $4 \mathrm{C})$. In summary, overexpression of miR-142-5p 

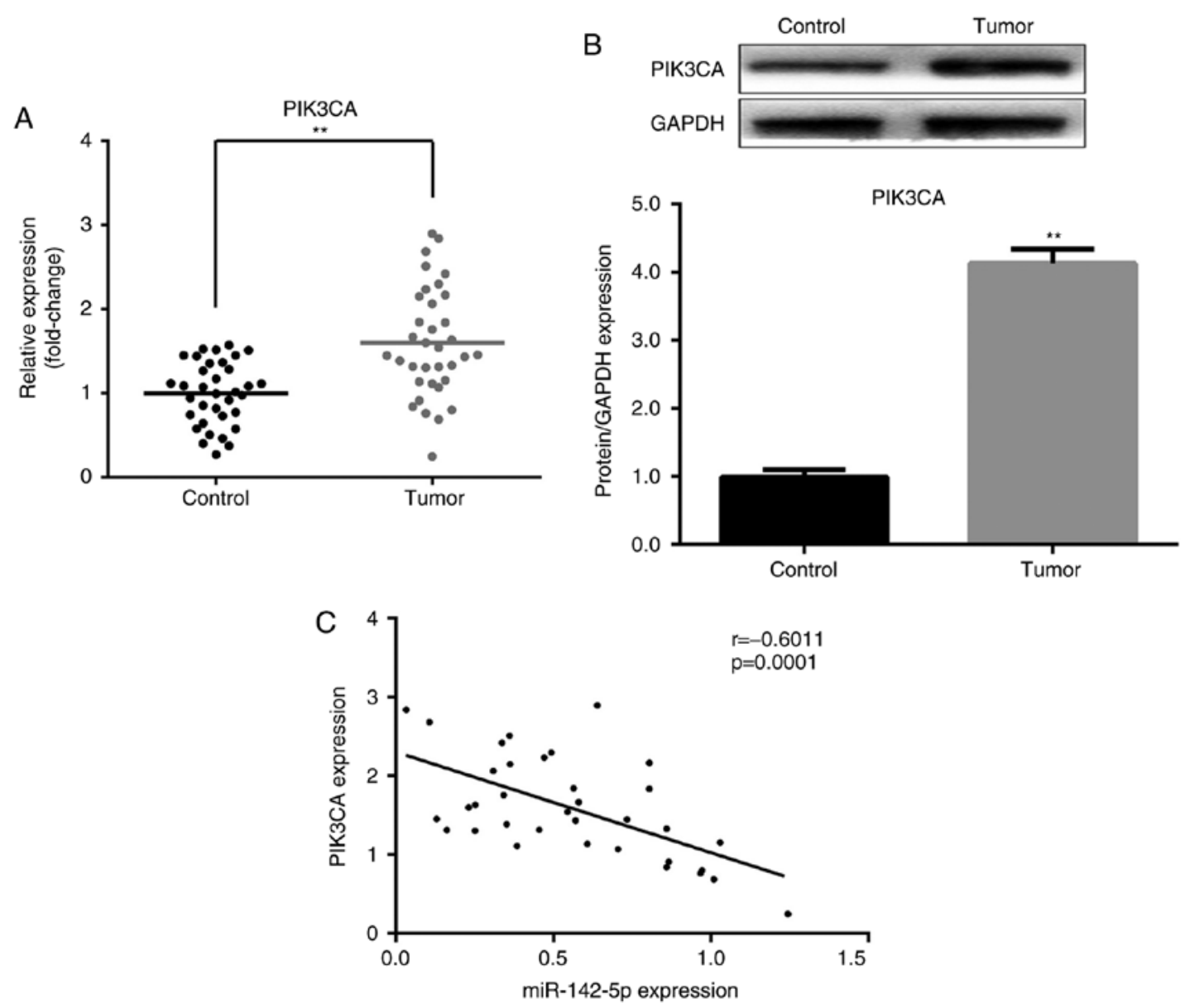

Figure 3. Expression of PIK3CA is increased in pancreatic tissues, and inversely correlated with miR-142-5p. (A) mRNA level of PIK3CA in cervical tissues and corresponding normal tissues was detected by reverse transcription-quantitative PCR. (B) Protein level of PIK3CA in tumor tissues and para-carcinoma tissues was measured by western blotting. GAPDH was used as an internal control. (C) Relationship between miR-142-5p and PIK3CA expression was evaluated by Spearman's correlation analysis; $\mathrm{r}=-0.6011, \mathrm{P}=0.0001$. Data are presented as the mean \pm standard error of the mean. ${ }^{* *} \mathrm{P}<0.01 \mathrm{vs}$. the control. PIK3CA, phosphoinositide-3-kinase catalytic subunit $\alpha$; miR, microRNA.

inhibited the proliferation, migration and invasion in pancreatic cancer cell by regulating PIK3CA.

miR-142-5p suppresses FAK and MMP9 expression, and the $P I 3 K / A K T$ pathway by targeting PIK3CA. Finally, the expression levels of FAK, MMP9, AKT and p-AKT were measured. As presented in Fig. 5A, B and D, overexpression of miR-142-5p significantly suppressed the mRNA expression of FAK and MMP9 ( $\mathrm{P}<0.01)$, while PIK3CA significantly reversed the suppression induced by miR-142-5p $(\mathrm{P}<0.01)$. Similar results were observed for protein expression $(\mathrm{P}<0.01)$. In addition, miR-142-5p-overexpression significantly reduced the protein level of p-AKT $(\mathrm{P}<0.01)$, while PIK3CA reversed the inhibition $(\mathrm{P}<0.01$; Fig. 5D). However, miR-142-5p and PIK3CA did not affect the expression of total AKT (Fig. 5C and D). These results suggested that miR-142-5p targets PIK3CA to suppress the expression of FAK and MMP9, as well as inactivate the PI3K/AKT signaling pathway.

\section{Discussion}

The present study revealed that miR-142-5p was downregulated in pancreatic cancer tissues and cell lines. PIK3CA was identified as a target of miR-142-5p. Notably, overexpression of miR-142-5p inhibited cell proliferation, migration and invasion of pancreatic cancer cells, suppressed the expression of FAK and MMP9, and inhibited the PI3K/AKT pathway by targeting PIK3CA.

miR-142-5p has been reported to be a critical miRNA during cancer progression (15). miR-142-5p functions as an oncogene or a tumor suppressor in different types of cancer. The expression of miR-142-5p is upregulated in renal cell carcinoma cells and overexpression of miR-142-5p promotes cell proliferation and migration (18). Similarly, miR-142-5p acts as an oncogene in colorectal cancer by enhancing the capacities of cell proliferation, colony formation and wound healing; however, it inhibits cell apoptosis $(19,20)$. Furthermore, miR-142-5p-overexpression suppresses the proliferation of non-small cell lung cancer (NSCLC) cells in vitro and in vivo, while inhibition of miR-142-5p promotes cell growth (21). miR-142-5p is also downregulated in hepatocellular carcinoma, which inhibits tumor cell viability, proliferation, migration and invasion, and induces apoptosis $(22,23)$. There are a limited number of studies regarding the functional role of miR-142-5p in pancreatic cancer cells. In the present study, miR-142-5p expression was revealed to be upregulated in pancreatic cancer tissues and cells. Furthermore, overexpression of miR-142-5p inhibited pancreatic cancer cell proliferation, migration and invasion. 


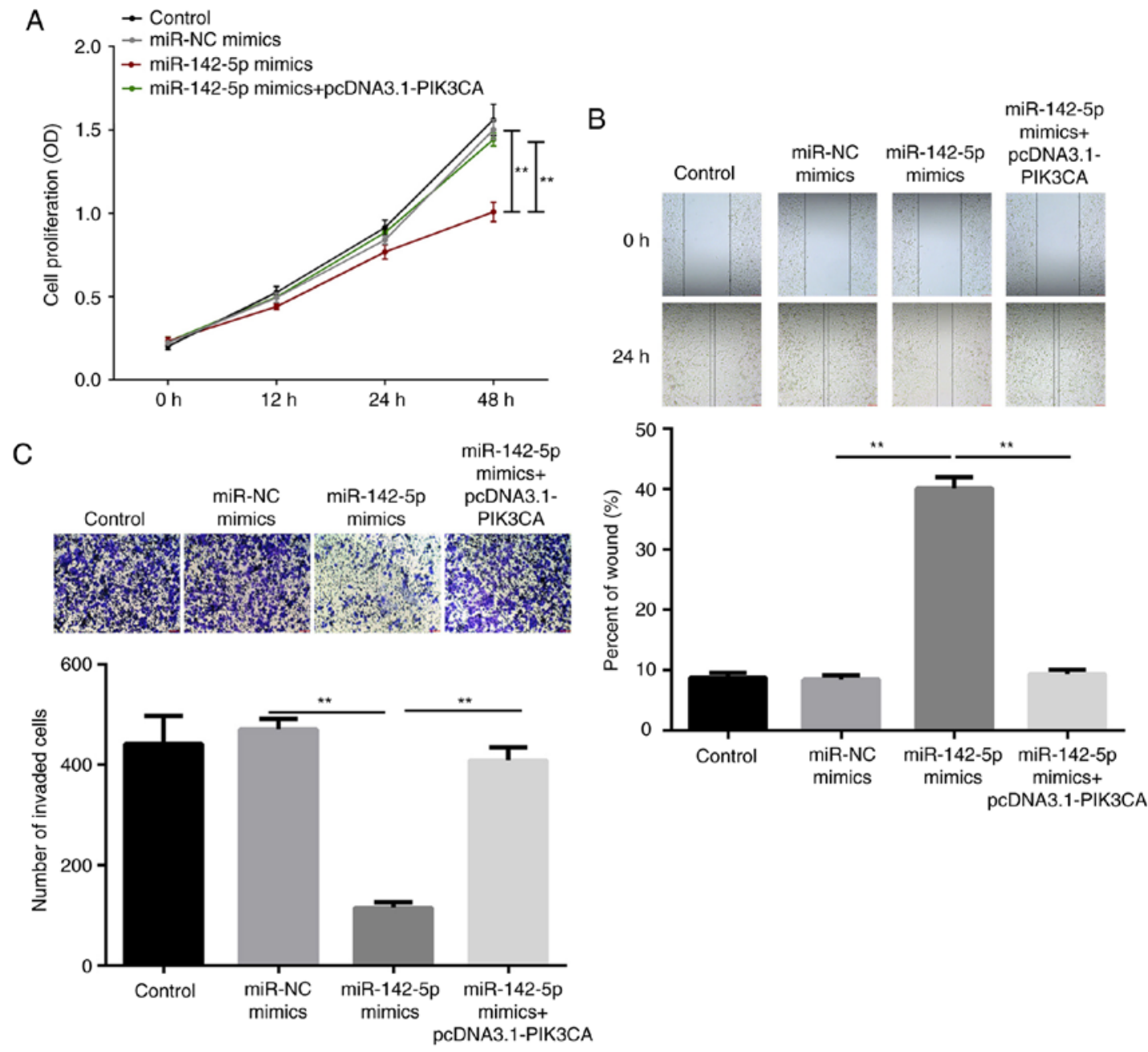

Figure 4. miR-142-5p inhibits proliferation, migration and invasion in PanC1 cells by targeting PIK3CA. (A) Cell proliferation was measured by Cell Counting Kit-8 after incubation of transfected PanC1 cells for 0, 12, 24 and $48 \mathrm{~h}$. (B) Wound healing assay was performed to detected migration ability of PanC1 cells after transfection for 0 and $24 \mathrm{~h}$ (magnification, $\mathrm{x} 100$ ). The percent of wound was quantified using a standard caliper. (C) Transwell assay of invasion of PanC1 cells after transfection for $24 \mathrm{~h}$ (magnification, $\mathrm{x} 200$ ). Number of invaded cells was quantified. Data are presented as the mean \pm standard error of the mean. ${ }^{* *} \mathrm{P}<0.01$. OD, optical density; miR, microRNA; NC, negative control; PIK3CA, phosphoinositide-3-kinase catalytic subunit $\alpha$.

These findings indicated that miR-142-5p functions as a tumor suppressor in pancreatic cancer. However, the underlying molecular mechanism requires further investigation.

A previous study reported that PIK3CA is a direct target of miR-142-5p in NSCLC (21). In the current study, bioinformatics analysis predicted that PIK3CA is a target of miR-142-5p and a dual-luciferase reporter assay confirmed this prediction. The PIK3CA gene is involved in encoding the catalytic subunit of PI3K P110a, which is activated by cell surface tyrosine kinase receptors $(24,25)$. PIK3CA often functions as an oncogene in different types of human cancer, including prostate cancer (26), lung cancer $(27,28)$, gastric cancer (29) and renal cell carcinoma (30). A previous study demonstrated that PIK3CA is an oncogene in pancreatic cancer (31). However, the biological role of PIK3CA remains unknown. In the present study, the expression of PIK3CA was upregulated in pancreatic cancer tissues and was negatively regulated by miR-142-5p expression in both tumor tissues and cells, which further verifies that PIK3CA is a target of miR-142-5p in pancreatic cancer. Furthermore, PIK3CA reversed the miR-142-5p-induced inhibition of cell proliferation, migration and invasion. These results demonstrated that miR-142-5p inhibits the proliferation, migration and invasion of pancreatic cancer cells by targeting PIK3CA.

FAK is a non-receptor tyrosine kinase that has been demonstrated to be upregulated in cancer and is involved in the progression of tumor aggressiveness (32). MMPs, including MMP9, have been reported to serve a role in cancer initiation, tumor growth and metastasis in pancreatic cancer (33). This study only focused on the expression of MMP9 rather than activity of MMP9, just as other miRNAs decrease cell migration and invasion through reducing MMP9 expression $(34,35)$. Additionally, PIK3CA regulates the activation of AKT and p-AKT activates various cellular processes $(36,37)$. In a previous study, knockdown of PIK3CA reduced FAK, MMP9 and p-AKT levels in glioblastoma multiforme cells (38). In the present study, miR-142-5p suppressed the expression of FAK, MMP9 and p-AKT, but did not affect AKT expression, while PIK3CA reversed the suppression. These findings suggested that miR-142-5p targets PIK3CA to suppress the expression of FAK and MMP9, and inactivate the PI3K/AKT signaling pathway. 

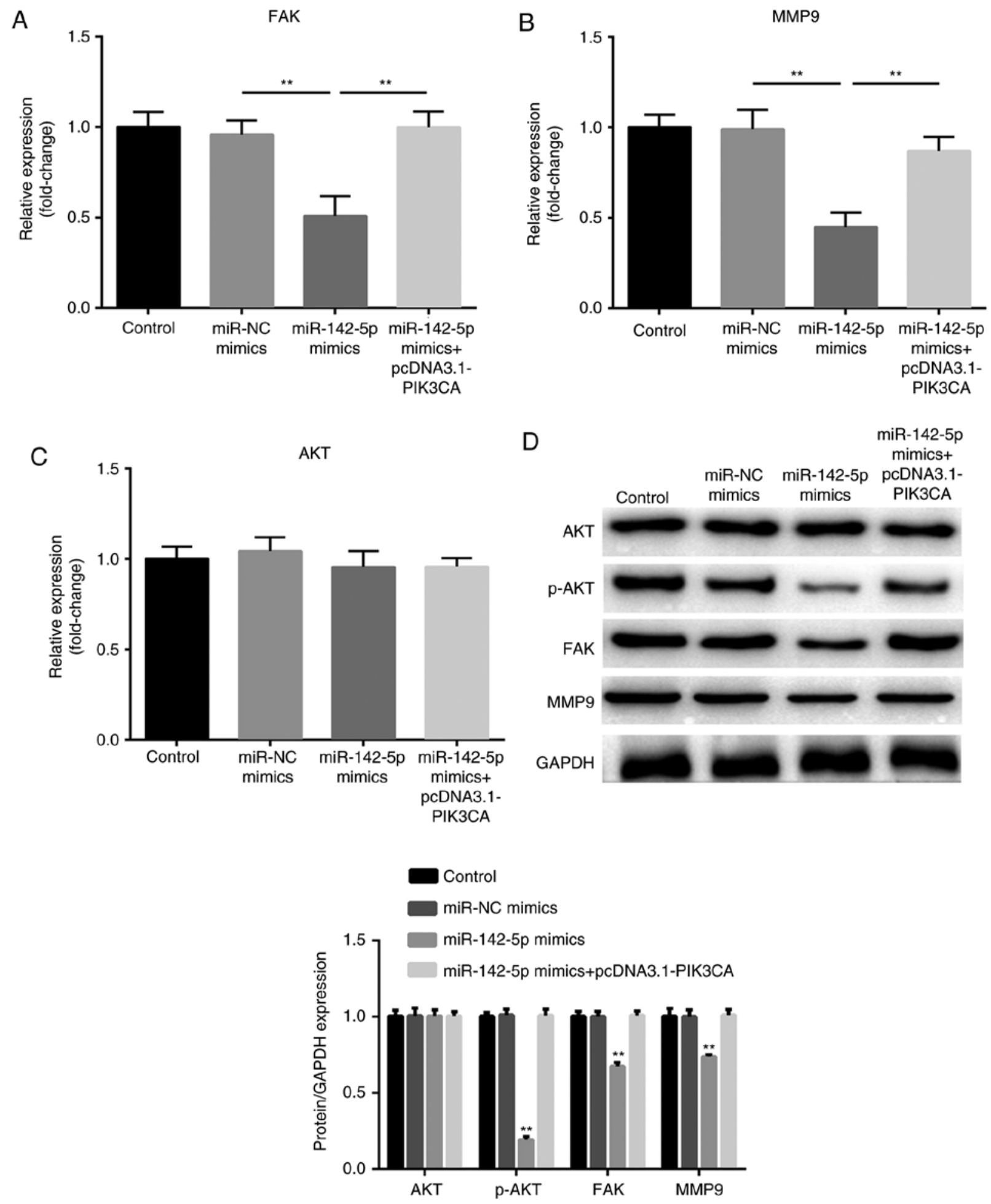

Figure 5. miR-142-5p suppresses the expression of FAK, MMP9 and inactivates the PI3K/AKT pathway through targeting PIK3CA. The expression of (A) FAK, (B) MMP9 and (C) AKT was detected by reverse transcription-quantitative PCR in transfected PanC1 cells. (D) Protein expression of FAK, MMP9, AKT and p-AKT was measured by western blotting. The relative protein expression, normalized for GAPDH, was quantified and shown in the corresponding histogram below. Data are presented as the mean \pm standard error of the mean. ${ }^{* *} \mathrm{P}<0.01 \mathrm{vs.} \mathrm{miR-NC}$ mimics. FAK, focal adhesion kinase; MMP, matrix metalloproteinase; PIK3CA, phosphoinositide-3-kinase catalytic subunit $\alpha$; AKT, protein kinase B; p-, phosphorylated-; NC, negative control.

In conclusion, the present study demonstrated that miR-142-5p functions as a tumor suppressor in pancreatic cancer, as it was identified to target PIK3CA and inhibit cell proliferation, migration and invasion in vitro by suppressing FAK and MMP9 expression, and inactivating the PI3K/AKT signaling pathway. These findings indicated that miR-142-5p may provide a novel target for the therapeutic treatment of pancreatic cancer.

\section{Acknowledgements}

Not applicable. 


\section{Funding}

No funding was received.

\section{Availability of data and materials}

All data generated or analyzed during this study are included in this published article.

\section{Authors' contributions}

JZ and YS contributed to study design. JZ, LZ, BW, ZQ, JW and $\mathrm{HH}$ performed experiments and data analysis. JZ was a major contributor in writing the manuscript. All authors have read and approved the final manuscript.

\section{Ethics approval and consent to participate}

The pancreatic cancer tissue and paired adjacent normal tissue sample collection was approved by the Ethics Committees of The Affiliated Huaian No. 1 People's Hospital of Nanjing Medical University. All patients provided written informed consent.

\section{Patient consent for publication}

Not applicable.

\section{Competing interests}

The authors declare they have no competing interests.

\section{References}

1. Kamisawa T, Wood LD, Itoi T and Takaori K: Pancreatic cancer. Lancet 388: 73-85, 2016.

2. Lin QJ, Yang F, Jin C and Fu DL: Current status and progress of pancreatic cancer in China. World J Gastroenterol 21: 7988-8003, 2015.

3. Goral V: Pancreatic cancer: Pathogenesis and diagnosis. Asian Pac J Cancer Prev 16: 5619-5624, 2015.

4. Siegel RL, Miller KD and Jemal A: Cancer statistics, 2016. CA Cancer J Clin 66: 7-30, 2016.

5. Carrato A, Falcone A, Ducreux M, Valle JW, Parnaby A, Djazouli K, Alnwick-Allu K, Hutchings A, Palaska C and Parthenaki I: A systematic review of the burden of pancreatic cancer in Europe: Real-world impact on survival, quality of life and costs. J Gastrointest Cancer 46: 201-211, 2015.

6. $\mathrm{Su} \mathrm{D}$, Yamaguchi $\mathrm{K}$ and Tanaka $\mathrm{M}$ : The characteristics of disseminated tumor cells in pancreatic cancer: A black box needs to be explored. Pancreatology 5: 316-324, 2005.

7. Li X, Li Y, Wan L, Chen R and Chen F: miR-509-5p inhibits cellular proliferation and migration via targeting MDM2 in pancreatic cancer cells. Onco Targets Ther 10: 4455-4464, 2017.

8. Stathis A and Moore MJ: Advanced pancreatic carcinoma: Current treatment and future challenges. Nat Rev Clin Oncol 7: $163-172,2010$.

9. Ambros V: microRNAs: Tiny regulators with great potential. Cell 107: 823-826, 2001.

10. Hammond SM: An overview of microRNAs. Adv Drug Deliv Rev 87: 3-14, 2015.

11. Bartel DP: MicroRNAs: Genomics, biogenesis, mechanism, and function. Cell 116: 281-297, 2004.

12. Shah MY, Ferrajoli A, Sood AK, Lopez-Berestein G and Calin GA: microRNA therapeutics in cancer-an emerging concept. EBioMedicine 12: 34-42, 2016.

13. Hata A and Lieberman J: Dysregulation of microRNA biogenesis and gene silencing in cancer. Sci Signal 8: re3, 2015.

14. Simonson B and Das S: MicroRNA therapeutics: The next magic bullet? Mini Rev Med Chem 15: 467-474, 2015.
15. Shrestha A, Mukhametshina RT, Taghizadeh S, Vasquez-PachecoE, Cabrera-Fuentes H, Rizvanov A, Mari B, Carraro G and Bellusci S: MicroRNA-142 is a multifaceted regulator in organogenesis, homeostasis, and disease. Dev Dynam 246: 285-290, 2017.

16. Jia L, Xi Q, Wang H, Zhang Z, Liu H, Cheng Y, Guo X, Zhang J, Zhang Q, Zhang L, et al: $\mathrm{miR}-142-5$ p regulates tumor cell PD-L1 expression and enhances anti-tumor immunity. Biochem Biophys Res Commun 488: 425-431, 2017.

17. Livak KJ and Schmittgen TD: Analysis of relative gene expression data using real-time quantitative PCR and the 2(-Delta Delta C(T)) method. Methods 25: 402-408, 2001.

18. Liu L, Liu S, Duan Q, Chen L, Wu T, Qian H, Yang S, Xin D, He Z and Guo Y: MicroRNA-142-5p promotes cell growth and migration in renal cell carcinoma by targeting BTG3. Am J Transl Res 9: 2394-2402, 2017.

19. Islam F, Gopalan V, Vider J, Lu CT and Lam AK: MiR-142-5p act as an oncogenic microRNA in colorectal cancer: Clinicopathological and functional insights. Exp Mol Pathol 104: 98-107, 2018.

20. Liu S, Xiao Z, Ai F, Liu F, Chen X, Cao K, Ren W, Zhang X, Shu P and Zhang D: miR-142-5p promotes development of colorectal cancer through targeting SDHB and facilitating generation of aerobic glycolysis. Biomed Pharmacother 92: 1119-1127, 2017.

21. Wang Z, Liu Z, Fang $X$ and Yang H: MiR-142-5p suppresses tumorigenesis by targeting PIK3CA in non-small cell lung cancer. Cell Physiol Biochem 43: 2505-2515, 2017.

22. Tsang FH, Au SL, Wei L, Fan DN, Lee JM, Wong CC, Ng IO and Wong CM: MicroRNA-142-3p and microRNA-142-5p are downregulated in hepatocellular carcinoma and exhibit synergistic effects on cell motility. Front Med 9: 331-343, 2015.

23. Lou K, Chen N, Li Z, Zhang B, Wang X, Chen Y, Xu H, Wang D and Wang H: MicroRNA-142-5p overexpression inhibits cell growth and induces apoptosis by regulating FOXO in hepatocellular carcinoma cells. Oncol Res 25: 65-73, 2017.

24. Samuels Y and Waldman T: Oncogenic mutations of PIK3CA in human cancers. Curr Top Microbiol Immunol 347: 21-41, 2010.

25. Vanhaesebroeck B, Stein RC and Waterfield MD: The study of phosphoinositide 3-kinase function. Cancer Surv 27: 249-270, 1996.

26. Zhang S, Cai J, Xie W, Luo H and Yang F: miR-202 suppresses prostate cancer growth and metastasis by targeting PIK3CA. Exp Ther Med 16: 1499-1504, 2018.

27. Meng $F$ and Zhang L: miR-183-5p functions as a tumor suppressor in lung cancer through PIK3CA inhibition. Exp Cell Res 374: 315-322, 2019.

28. Yu QQ, Wu H, Huang X, Shen H, Shu YQ, Zhang B, Xiang CC, Yu SM, Guo RH and Chen L: MiR-1 targets PIK3CA and inhibits tumorigenic properties of A549 cells. Biomed Pharmacother 68: 155-161, 2014.

29. Liang M, Shi B, Liu J, He L, Yi G, Zhou L, Yu G and Zhou X: Downregulation of miR203 induces overexpression of PIK3CA and predicts poor prognosis of gastric cancer patients. Drug Des Devel Ther 9: 3607-3616, 2015.

30. Chen K, Zeng J, Tang K, Xiao H, Hu J, Huang C, Yao W, Yu G, Xiao W, Guan W, et al: miR-490-5p suppresses tumour growth in renal cell carcinoma through targeting PIK3CA. Biol Cell 108: 41-50, 2016.

31. Waddell N, Pajic M, Patch AM, Chang DK, Kassahn KS, Bailey P, Johns AL, Miller D, Nones K, Quek K, et al: Whole genomes redefine the mutational landscape of pancreatic cancer. Nature 518: 495-501, 2015.

32. Lv PC, Jiang AQ, Zhang WM and Zhu HL: FAK inhibitors in Cancer, a patent review. Expert Opin Ther Pat 28: 139-145, 2018.

33. Knapinska AM, Estrada CA and Fields GB: The roles of matrix metalloproteinases in pancreatic cancer. Prog Mol Biol Transl Sci 148: 339-354, 2017.

34. Xu X, Gao F, Wang J, Tao L, Ye J, Ding L, Ji W and Chen X: MiR-122-5p inhibits cell migration and invasion in gastric cancer by down-regulating DUSP4. Cancer Biol Ther 19: 427-435, 2018.

35. Zou Q, Yi W, Huang J, Fu F, Chen G and Zhong D: MicroRNA-375 targets PAX6 and inhibits the viability, migration and invasion of human breast cancer MCF-7 cells. Exp Ther Med 14: 1198-1204, 2017.

36. Osaki M, Oshimura M and Ito H: PI3K-Akt pathway: Its functions and alterations in human cancer. Apoptosis 9: 667-676, 2004.

37. Vivanco I and Sawyers CL: The phosphatidylinositol 3-Kinase AKT pathway in human cancer. Nat Rev Cancer 2: 489-501, 2002.

38. Weber GL, Parat MO, Binder ZA, Gallia GL and Riggins GJ: Abrogation of PIK3CA or PIK3R1 reduces proliferation, migration, and invasion in glioblastoma multiforme cells. Oncotarget 2: 833-849, 2011. 